\title{
The Objective Use of Pulse Oximetry to Predict Respiratory Support Transition in Preterm Infants: An Observational Pilot Study
}

\author{
Kemi K Mascoll-Robertson MBBS, Rose M Viscardi MD, and Hyung C Woo MD
}

\begin{abstract}
BACKGROUND: Preterm infants often require some form of respiratory support with supplemental oxygen and are monitored by continuous pulse oximetry $\left(\mathrm{S}_{\mathrm{pO}_{2}}\right)$. The study objective was to determine whether the histogram distribution of $\mathrm{S}_{\mathrm{pO}_{2}}$ over a 24-h period will predict readiness for weaning respiratory support in preterm infants. We hypothesize that infants with $\geq 15 \%$ of time spent with $\mathrm{S}_{\mathrm{pO}_{2}}<86 \%$ before transitioning from CPAP or high-flow nasal cannula (HFNC) to low-flow nasal cannula, oxyhood, or room air are more likely to fail transitioning. METHODS: The $\mathrm{S}_{\mathrm{pO}_{2}}$ histograms were downloaded daily for 31 infants, 24-32 weeks gestational age, before transition from CPAP or HFNC to low-flow nasal cannula, oxyhood, or room air. The $\mathrm{S}_{\mathrm{pO}_{2}}$ histogram downloads were continued for each infant for 1 week after transition or when the infant reached 36 weeks postmenstrual age or when $\mathrm{S}_{\mathrm{pO}_{2}}$ monitoring was discontinued. Failure was defined as an increase in respiratory support within $72 \mathrm{~h}$ of transitioning. We compared the percentage of time for each $\mathrm{S}_{\mathrm{pO}_{2}}$ quintile for the 24-h periods before and immediately following CPAP/HFNC transitioning between groups. RESULTS: Twenty-four subjects transitioned successfully, but 7 subjects failed. Two of 7 subjects (28.6\%) who failed transition experienced $\mathrm{S}_{\mathrm{pO}_{2}}<86 \% \geq 15 \%$ of the time pretransition compared with none in the success group $(P=.045)$. The failure group experienced $\mathrm{S}_{\mathrm{pO}_{2}}<86 \% 10.7 \pm 11.9 \%$ of time pre-wean compared with $3.3 \pm 4.7 \%$ of time in the success group $(P \stackrel{.02}{=}$. In contrast, infants who were successfully weaned tended to experience a greater percentage of time with $\mathrm{S}_{\mathrm{pO}_{2}}>95 \%$ compared with the failure group, both pre-wean $(54.3 \pm 36.1 \%$ vs $33 \pm 27.7 \%, P=.16)$ and post-wean $(52 \pm 35.4 \%$ vs $27.4 \pm 27.7 \%, P=.10)$. CONCLUSIONS: These data suggest that pulse oximetry histograms may be useful in assessing CPAP/HFNC support transition readiness. Key words: pulse oximetry; SpO2; histogram; respiratory support weaning; prematurity; CPAP; high-flow nasal cannula; HFNC. [Respir Care 2016;61(4):416-422. (C 2016 Daedalus Enterprises]
\end{abstract}

\section{Introduction}

Preterm infants often require some form of respiratory support with supplemental oxygen. Regulating oxygen exposure is essential because hyperoxia is associated with

The authors are affiliated with the Department of Pediatrics, University of Maryland School of Medicine, Baltimore, Maryland.

Dr Mascoll-Robertson presented a version of this paper at the Pediatric Academic Societies/Society for Pediatric Research Annual Meeting, held May 4-7, 2013, in Washington, DC.

The authors have disclosed no conflicts of interest.

Correspondence: Hyung Chul Woo MD, University of Maryland School of Medicine, 110 South Paca Street, 8th Floor, Baltimore, MD 21201.

E-mail: hwoo@peds.umaryland.edu.

DOI: $10.4187 /$ respcare.04102 retinopathy of prematurity and bronchopulmonary dysplasia, ${ }^{1,2}$ whereas intermittent hypoxia is associated with increased mortality, severe retinopathy of prematurity, and pulmonary hypertension. ${ }^{2-7}$ Continuous pulse oximetry $\left(\mathrm{S}_{\mathrm{pO}_{2}}\right)$ measured by new generation high-resolution pulse oximeters is more accurate than hand-transcribed $\mathrm{S}_{\mathrm{pO}_{2}}$ values, and more episodes of intermittent hypoxia are detected. ${ }^{89}$ Hence, high-resolution pulse oximetry with target $\mathrm{O}_{2}$ ranges has been implemented in many neonatal ICUs to avoid fluctuations in $\mathrm{S}_{\mathrm{pO}_{2}}$ and to reduce the risk of adverse outcomes. ${ }^{10-12}$ Despite these advances, maintaining $\mathrm{S}_{\mathrm{pO}_{2}}$ within the targeted range is difficult. ${ }^{13-17}$

To reduce lung injury from mechanical ventilation, noninvasive modes of respiratory support, such as nasal CPAP and high-flow nasal cannula (HFNC), have been increasingly used. ${ }^{18-23}$ However, there is no agreement on best practices for weaning these devices. ${ }^{24-27}$ Noninvasive in- 
dicators demonstrating readiness for reducing respiratory support are lacking. As a first step to develop a tool to assess readiness for transition from noninvasive respiratory support, we conducted an observational study to determine whether the pulse oximetry histogram distribution is an objective noninvasive tool to predict readiness for change in respiratory support in preterm infants and avoid $\mathrm{S}_{\mathrm{pO}_{2}}$ fluctuations that can be exacerbated during the transition period. We hypothesized that infants with $\geq 15 \%$ of cumulative time spent with $\mathrm{S}_{\mathrm{pO}_{2}}<86 \%$ in the 24-h period before transitioning from CPAP or HFNC to low-flow nasal cannula, oxyhood, or room air are more likely to fail transition.

\section{See the Related Editorial on Page 569}

\section{Methods}

This was a prospective, observational cohort study conducted at the University of Maryland Medical Center neonatal ICU from April 2012 to July 2013. The University of Maryland institutional review board approved the study protocol, and parental consent was obtained for each subject. Inclusion criteria were (1) gestational ages 24-32 weeks and (2) receipt of CPAP or HFNC ( $\geq 2 \mathrm{~L} / \mathrm{min}$ ) for at least $24 \mathrm{~h}$. Exclusion criteria were (1) positive pressure respiratory support other than CPAP/HFNC; (2) congenital heart disease or pulmonary anomalies; and (3) persistent pulmonary hypertension. Infants who had been receiving mechanical ventilation support were screened for eligibility once they were extubated to CPAP or HFNC.

Respiratory management of all study infants was provided according to the University of Maryland Medical Center neonatal ICU guidelines for criteria for mechanical ventilation, extubation, CPAP and HFNC initiation, and low-flow nasal cannula and oxyhood use. Mechanical ventilation is instituted for respiratory failure $\left(\mathrm{P}_{\mathrm{aO}}<50\right.$ with $\mathrm{F}_{\mathrm{IO}_{2}} \geq 0.60$ and/or $\mathrm{P}_{\mathrm{aCO}_{2}}>55$ ), and extubation to CPAP or $\mathrm{HFNC}$ is considered once positive inspiratory pressure is $\leq 18 \mathrm{~cm} \mathrm{H}_{2} \mathrm{O}$, PEEP is $4-5 \mathrm{~cm} \mathrm{H}_{2} \mathrm{O}$, the intermittent mandatory ventilation rate is $\leq 15$, and the $\mathrm{F}_{\mathrm{IO}_{2}}$ is $<0.3$. CPAP or HFNC is used as the primary mode of noninvasive respiratory support if $\mathrm{P}_{\mathrm{aCO}_{2}}$ is $<55 \mathrm{~mm} \mathrm{Hg}, \mathrm{F}_{\mathrm{IO}_{2}}$ is $<0.6$, and $<6$ apnea/bradycardia/desaturation episodes occur within $6 \mathrm{~h}$ requiring stimulation. Transition from CPAP or HFNC is considered once the infant reaches CPAP of 4-5 $\mathrm{cm} \mathrm{H}_{2} \mathrm{O}$ or $\mathrm{HFNC}$ of $2 \mathrm{~L} / \mathrm{min}, \mathrm{F}_{\mathrm{IO}_{2}}<0.3$ and not increasing, no significant chest retractions, and infrequent apnea/bradycardia/desaturation episodes. It is our neonatal ICU practice to transition off CPAP or HFNC with the intention to remain off support. CPAP support was provided by the VIASYS Infant Flow SiPAP System (CareFusion, San Diego, California) and HFNC by Precision Flow (Vapotherm, Exeter, New Hampshire).

\section{QUICK LOOK}

\section{Current knowledge}

To reduce lung injury from mechanical ventilation in preterm infants, noninvasive modes of respiratory support, such as nasal CPAP and high-flow nasal cannula, have been increasingly used. Noninvasive indicators demonstrating readiness for weaning from noninvasive respiratory support are lacking.

\section{What this paper contributes to our knowledge}

In a pilot study of preterm infants $\leq 32$ weeks gestation receiving CPAP or high-flow nasal cannula, the percentage of time with $\mathrm{S}_{\mathrm{pO}_{2}}<86 \%$ in the $24 \mathrm{~h}$ preceding transition was greater in infants who failed transition compared with those who were successfully weaned off noninvasive respiratory support. Infants who were successfully transitioned tended to experience a greater percentage of time with $\mathrm{S}_{\mathrm{pO}_{2}}>95 \%$ compared with the failure group, both pre- and post-wean.

For the study, the attached Nellcor OxiMax N-600x pulse oximeter (Covidien, Mansfield, Massachusetts) was set to both neonatal and normal modes, where data were gathered every $4 \mathrm{~s}$ with an averaging time of 5-7 s. The target oxygen saturation range used was set by the current University of Maryland Medical Center neonatal ICU postmenstrual age-based targeted oxygen saturation protocol, where targeted $\mathrm{S}_{\mathrm{pO}_{2}}$ is $88-93 \%$ for infants $<33$ weeks postmenstrual age and $90-97 \%$ for infants $\geq 33$ weeks postmenstrual age. The histogram distribution of pulse oximetry displaying the percentage of time spent in the pulse oximeter preset oxygen saturation ranges (96-100, 91-95, $86-90,81-85$, and $<80 \%$ ) for a $24-\mathrm{h}$ period was recorded daily until the infants were transitioned to low-flow nasal cannula $(\leq 1.5 \mathrm{~L} / \mathrm{min})$, oxyhood, or room air for 1 week post-transition or until the infant reached 36 weeks postmenstrual age without attempted transition. The primary outcome was transition success defined as transition to low-flow nasal cannula, room air, or oxyhood for $72 \mathrm{~h}$. If a subject was restarted on CPAP or HFNC by the clinical team within $72 \mathrm{~h}$ after transitioning to low-flow nasal cannula/oxyhood/room air, the subject was considered a transition failure. The clinical team was blinded to the 24-h histogram distributions, and these data were not incorporated into clinical decision-making. The clinical team made all respiratory care decisions concerning timing of CPAP/HFNC transition to lower respiratory support as well as subsequent decisions to increase respiratory support post-transition based on neonatal ICU guidelines.

Data collection included demographic and clinical variables, such as presence of patent ductus arteriosus; ante- 
natal steroids; surfactant administration; average percentage of $\mathrm{F}_{\mathrm{IO}_{2}}$ over 24-h intervals; documented apnea, bradycardia, and/or desaturation events; and clinical outcomes, such as retinopathy of prematurity, necrotizing enterocolitis, intraventricular hemorrhage, and bronchopulmonary dysplasia defined as receipt of supplemental oxygen or positive pressure support at 36 weeks postmenstrual age. For infants receiving low-flow nasal cannula, the effective $\mathrm{F}_{\mathrm{IO}_{2}}$ was calculated using the STOP-ROP tables. ${ }^{28}$

The sample size of 30 subjects for the primary outcome was chosen in order to provide adequate numbers to determine whether the $\mathrm{S}_{\mathrm{pO}_{2}}$ histogram pattern for babies who fail transition from CPAP/HFNC to low-flow nasal cannula/oxyhood/room air differs from the $\mathrm{S}_{\mathrm{pO}_{2}}$ histogram pattern of infants who make a successful transition. We selected $<86 \% \mathrm{~S}_{\mathrm{pO}_{2}}$ as a clinically important level of hypoxemia. Since studies of high-resolution oximetry indicate that $\mathrm{S}_{\mathrm{pO}_{2}}$ in the hypoxemic range occurs $<15 \%$ of the time during noninvasive respiratory support with active $\mathrm{O}_{2}$ target range management, ${ }^{8,29}$ we selected a cut-off of $\geq 15 \%$ of time with $\mathrm{S}_{\mathrm{pO}_{2}}<86 \%$ as high-risk for transition failure. In a preliminary sample of 5 infants $<33$ weeks postmenstrual age at our neonatal ICU, 2 infants (40\%) experienced $\mathrm{S}_{\mathrm{pO}_{2}}<86 \%>15 \%$ of the time during the transition from CPAP to low-flow nasal cannula (unpublished data). For the sample size, we assumed that one third of the subjects would have $\geq 15 \%$ of time spent with $\mathrm{S}_{\mathrm{pO}_{2}}<86 \%$ when transitioning off CPAP/HFNC and that $75 \%$ of those babies would fail transitioning. With these assumptions, a sample size of 30 was determined to be sufficient with a power $=0.848$ and an $\alpha=0.05$ to detect a difference if the failure rate for infants who have $\leq 15 \%$ of time with $\mathrm{S}_{\mathrm{pO}_{2}}<86 \%$ is $15 \%$.

The mean $\pm \mathrm{SD}$ of the percentage of time for each $\mathrm{S}_{\mathrm{pO}_{2}}$ quintile over time for each subject was calculated for each period of respiratory support. The percentage of time for each $\mathrm{S}_{\mathrm{pO}_{2}}$ quintile for the 24-h period pre- and post-transition from CPAP/HFNC to low-flow nasal cannula/oxyhood/room air was compared between the successfully transitioned and transition failure groups. Demographic and respiratory variables were compared between the groups by chi-square test for categorical variables and $t$ test for continuous variables. Receiver operating characteristic curve analysis was done to assess the sensitivity and specificity of different percentage of time cut-offs for $\mathrm{S}_{\mathrm{pO}_{2}}$ $<86 \%$. The Spearman's rank correlation test was used to calculate the correlation coefficient between percent change in $\mathrm{F}_{\mathrm{IO}_{2}}$ and the percentage of time with $\mathrm{S}_{\mathrm{pO}_{2}}<86 \%$ and $\mathrm{S}_{\mathrm{pO}_{2}}>95 \%$ for the 24 -h periods pre- and post-transition.

\section{Results}

Of 137 infants admitted to the University of Maryland Medical Center neonatal ICU with gestational ages of

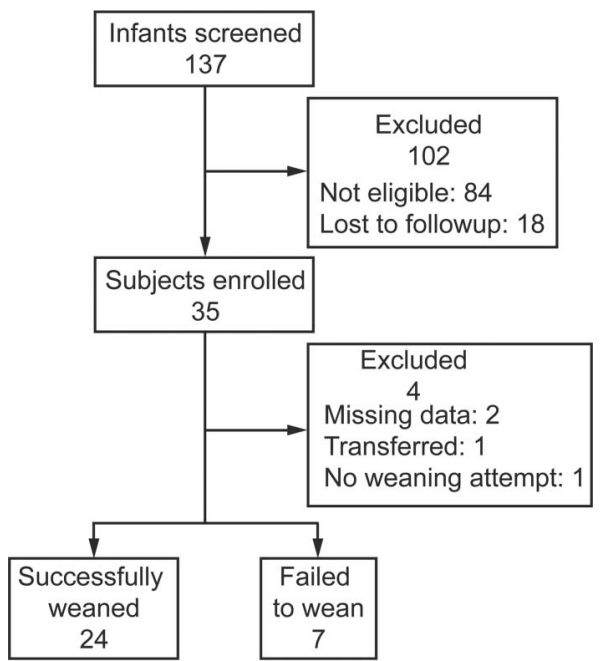

Fig. 1. Flow chart. Weaning success was defined as weaning to low-flow nasal cannula, room air, or oxygen hood for $7 \mathrm{~d}$. Weaning failure was defined as returning to CPAP or high-flow nasal cannula within $72 \mathrm{~h}$.

24-32 weeks, 53 were eligible for the study, and consent was obtained from the 35 eligible subjects who were approached for consent (Fig. 1). Eighteen eligible subjects were missed due to unavailability of the investigators. Four subjects were not included in the final analysis due to missing data $(n=2)$, transfer to another facility before transition $(n=1)$, and no transition attempt before 36 weeks postmenstrual age $(n=1)$. Seven of the 31 subjects were $\geq 33$ weeks postmenstrual age at the time of transition. Twenty-four subjects transitioned successfully from CPAP/HFNC, but 7 subjects failed transition. In all cases of failed transition, CPAP was reinstituted due to increased work of breathing assessed as an increased severity of subcostal and intercostal retractions, nasal flaring, and poor air entry on auscultation. All 7 failed subjects transitioned from CPAP; 6 infants were transitioned from CPAP $+4 \mathrm{~cm} \mathrm{H}_{2} \mathrm{O}$, and 1 infant was transitioned from CPAP $+5 \mathrm{~cm} \mathrm{H}_{2} \mathrm{O}$. There were a total of 8 subjects transitioned from HFNC, all in the success group, 7 infants transitioned from $2 \mathrm{~L} / \mathrm{min}$ and 1 from $3 \mathrm{~L} / \mathrm{min}$. There were 16 babies who transitioned successfully from CPAP, 14 infants transitioned from $\mathrm{CPAP}+4 \mathrm{~cm} \mathrm{H}_{2} \mathrm{O}$, and 2 babies from CPAP $+5 \mathrm{~cm} \mathrm{H}_{2} \mathrm{O}$. The failed transition subjects were less mature but had similar birth weights compared with the successful group (Table 1). The groups were similar for Apgar scores, exposure to antenatal steroids, surfactant, and patent ductus arteriosus treated with either indomethacin or surgical ligation. Both groups experienced similar rates of retinopathy of prematurity of at least Stage 2 , intraventricular hemorrhage, and bronchopulmonary dysplasia at 36 weeks postmenstrual age.

At the time of CPAP/HFNC transition, there was no significant difference in postmenstrual age or body weight 
between the groups (Table 2). Two of the 7 subjects (29\%) in the failure group experienced $\mathrm{S}_{\mathrm{pO}_{2}}<86 \% \geq 15 \%$ of the time pre-wean compared with none in the successful group $(P=.045)$. The failure group experienced $\mathrm{S}_{\mathrm{pO}_{2}}<86 \%$

Table 1. Characteristics of Study Subjects

\begin{tabular}{lccc}
\hline \hline \multicolumn{1}{c}{ Variable } & $\begin{array}{c}\text { Transition } \\
\text { Success } \\
(n=24)\end{array}$ & $\begin{array}{c}\text { Transition } \\
\text { Failure } \\
(n=7)\end{array}$ & $P$ \\
\hline Gestational age, mean \pm SD wks & $28.5 \pm 2.0$ & $26.6 \pm 2.0$ & .04 \\
Birth weight, mean \pm SD g & $1168 \pm 282$ & $973 \pm 323$ & .13 \\
Male sex, $n(\%)$ & $17(71)$ & $4(57)$ & .49 \\
Race, $n(\%)$ & & & .78 \\
Caucasian & $12(50)$ & $3(43)$ & \\
African-American & $11(46)$ & $4(57)$ & \\
Hispanic & $1(4)$ & $0(0)$ & \\
Other & $0(0)$ & $0(0)$ & \\
Antenatal steroids, $n(\%)$ & $22(92)$ & $7(100)$ & .43 \\
Surfactant, $n(\%)$ & $14(58)$ & $6(85)$ & .18 \\
PDA, $n(\%)$ & $5(21)$ & $2(29)$ & .66 \\
Apgar score 1 min, mean \pm SD & $5.1 \pm 2.5$ & $5.3 \pm 2.3$ & .88 \\
Apgar score 5 min, mean \pm SD & $7.1 \pm 2.3$ & $6.9 \pm 2.7$ & .79 \\
IVH, $n(\%)$ & $2(8)$ & $2(29)$ & .16 \\
ROP stage $\geq 2, n(\%)$ & $1(4)$ & $1(14)$ & .49 \\
NEC stage $\geq 2, n(\%)$ & $1(4)$ & $1(14)$ & .34 \\
BPD at 36 weeks PMA, $n(\%)$ & $6(25)$ & $0(0)$ & .14 \\
& & & \\
\hline PDA $=$ patent ductus arteriosus treated with indomethacin or surgical ligation & \\
IVH $=$ intraventricular hemorrhage & & & \\
ROP $=$ retinopathy of prematurity & & & \\
NEC $=$ necrotizing enterocolitis & & & \\
PPD $=$ bronchopulmonary dysplasia & & & \\
& & & \\
\hline
\end{tabular}

$11 \pm 12 \%$ of the time pre-wean compared with $3 \pm 5 \%$ of time in the successful group $(P=.02)$ (Fig. 2). A receiver operating characteristic curve was generated, revealing that $\mathrm{S}_{\mathrm{pO}_{2}}<86 \% \geq 15 \%$ of time had $29 \%$ sensitivity and $96 \%$ specificity for wean failure, whereas a cut-off of $\geq 10 \%$ of time had $43 \%$ sensitivity and $83 \%$ specificity. Infants who were successfully transitioned tended to experience a greater percentage of time with $\mathrm{S}_{\mathrm{pO}_{2}}>95 \%$ compared with the failure group, both pre-wean $(54 \pm 36$ vs $33 \pm 28 \%$, $P=.16)$ and post-wean $(52 \pm 35$ vs $27 \pm 28 \%, P=.10)$ (Fig. 2). There was no difference in effective $\mathrm{F}_{\mathrm{IO}_{2}}$ or breathing frequency between the successful and wean failure groups both pre- and post-wean (Table 2). In addition, there was no statistical difference in $\mathrm{S}_{\mathrm{pO}_{2}}$ histogram distribution between CPAP or HFNC subjects pre- or postwean (Fig. 3) and between infants $<33$ weeks and $\geq 33$ weeks postmenstrual age pre- or post-wean (data not shown). However, as the percentage of time $\mathrm{S}_{\mathrm{pO}_{2}}<86 \%$ pre-transition increased, the percentage change in effective $\mathrm{F}_{\mathrm{IO}_{2}}$ post-transition increased $(\rho=0.585, P=.01)$ (Fig. $4 \mathrm{~A})$. Conversely, as the percentage of time $\mathrm{S}_{\mathrm{pO}_{2}}>95 \%$ pre-transition increased, the percentage change in the effective $\mathrm{F}_{\mathrm{IO}_{2}}$ decreased $(\rho=-0.431, P=.035)$ (Fig. 4B).

\section{Discussion}

Noninvasive respiratory support is commonly used in the management of respiratory distress even in the smallest infants. Recent randomized control trials have indicated that early CPAP may be an effective alternative to prophylactic surfactant administration. ${ }^{18,20,21,23}$ When

Table 2. Comparison of Respiratory Variables in Success and Failure Groups Pre- and Post-Transition

\begin{tabular}{|c|c|c|c|}
\hline Variable & Transition Success $(n=24)$ & Transition Failure $(n=7)$ & $P$ \\
\hline Corrected GA at time of transition, mean $\pm \mathrm{SD}$ wks & $31.2 \pm 2.2$ & $30.3 \pm 1.6$ & .39 \\
\hline Postnatal age at time of transition, mean \pm SD wks & $19.7 \pm 18.6$ & $25 \pm 20.4$ & .52 \\
\hline Body weight at time of transition, mean $\pm \mathrm{SD} g$ & $1358 \pm 386$ & $1167 \pm 256$ & .23 \\
\hline Duration of IMV pre-transition, mean $\pm \mathrm{SD} d$ & $7 \pm 12$ & $8 \pm 9$ & .86 \\
\hline Duration of CPAP/HFNC pre-transition, mean $\pm \mathrm{SD} d$ & $13 \pm 14$ & $17 \pm 13$ & .46 \\
\hline \multicolumn{4}{|l|}{ Respiratory support pre-transition, $n(\%)$} \\
\hline CPAP & $16(67)$ & $7(100)$ & .08 \\
\hline HFNC & $8(33)$ & $0(0)$ & \\
\hline \multicolumn{4}{|l|}{ Respiratory support post-transition, $n(\%)$} \\
\hline Low-flow nasal cannula & $19(79)$ & $7(100)$ & .19 \\
\hline Room air & $5(21)$ & $0(0)$ & \\
\hline Percent $\mathrm{S}_{\mathrm{pO}_{2}}<86 \% \geq 15 \%$ of time pre-transition & $0(0)$ & $2(28.6)$ & .045 \\
\hline Effective $\mathrm{F}_{\mathrm{IO}_{2}}$ pre-transition, median (range) & $0.21(0.21-0.23)$ & $0.22(0.21-0.37)$ & .75 \\
\hline Effective $\mathrm{F}_{\mathrm{IO}_{2}}$ post-transition, median (range) & $0.21(0.21-0.48)$ & $0.25(0.21-0.31)$ & .28 \\
\hline Breathing frequency pre-transition, mean \pm SD breaths/min & $54 \pm 6$ & $52 \pm 6$ & .36 \\
\hline Breathing frequency post-transition, mean $\pm \mathrm{SD}$ breaths/min & $59 \pm 8$ & $58 \pm 7$ & .81 \\
\hline \multicolumn{4}{|l|}{$\begin{array}{l}\text { GA }=\text { gestational age } \\
\text { IMV = intermittent mandatory ventilation } \\
\text { HFNC = high-flow nasal cannula }\end{array}$} \\
\hline
\end{tabular}



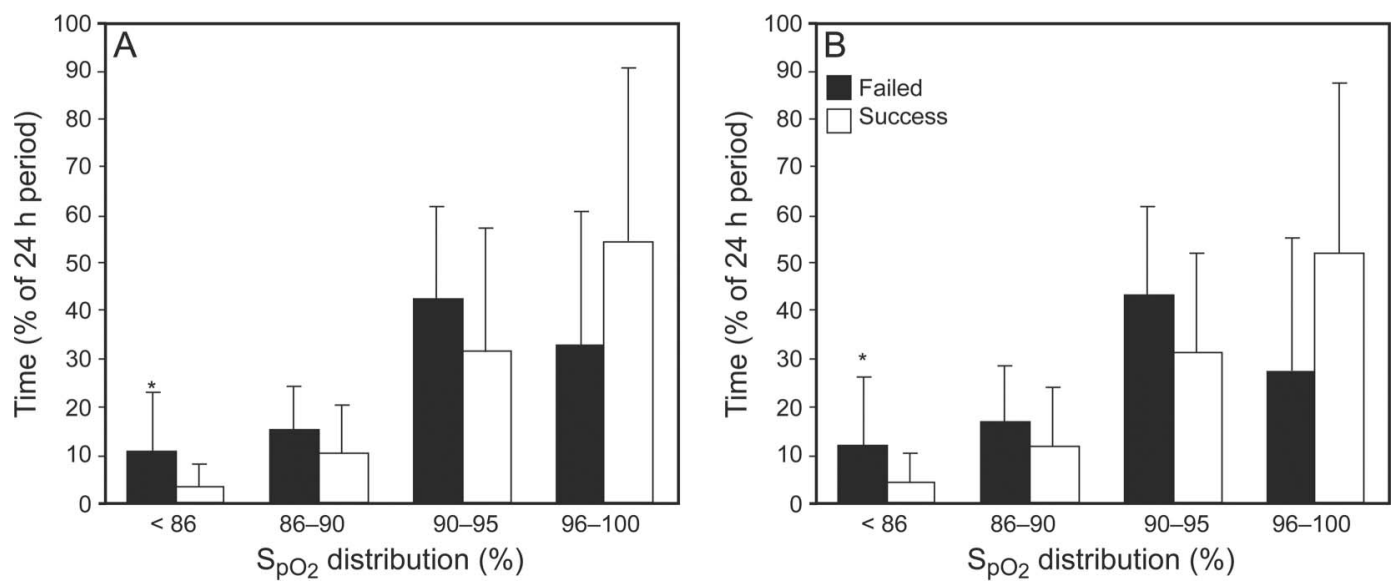

Fig. 2. Comparison of $\mathrm{S}_{\mathrm{pO}_{2}}$ histogram distribution pre-transition (A) and post-transition (B) between successful and failed transition groups. $\mathrm{S}_{\mathrm{pO}_{2}}$ histogram was recorded for the 24-h periods pre- and post-transition from either CPAP or high-frequency nasal cannula to lowfrequency nasal cannula or room air. Data are expressed as mean $\pm \mathrm{SD}$. * $P<.05$.
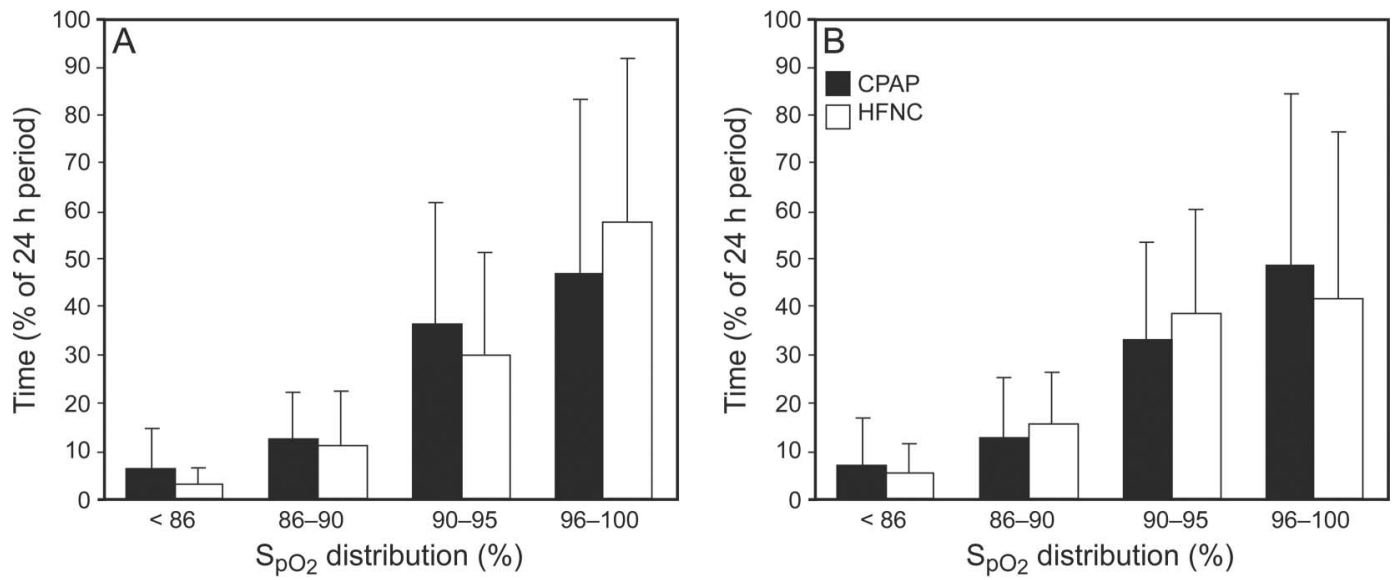

Fig. 3. Comparison of $\mathrm{S}_{\mathrm{pO}_{2}}$ histogram distribution pre-transition (A) and post-transition (B) of subjects transitioned from CPAP or highfrequency nasal cannula (HFNC) to low-frequency nasal cannula or room air. Data are expressed as mean \pm SD.
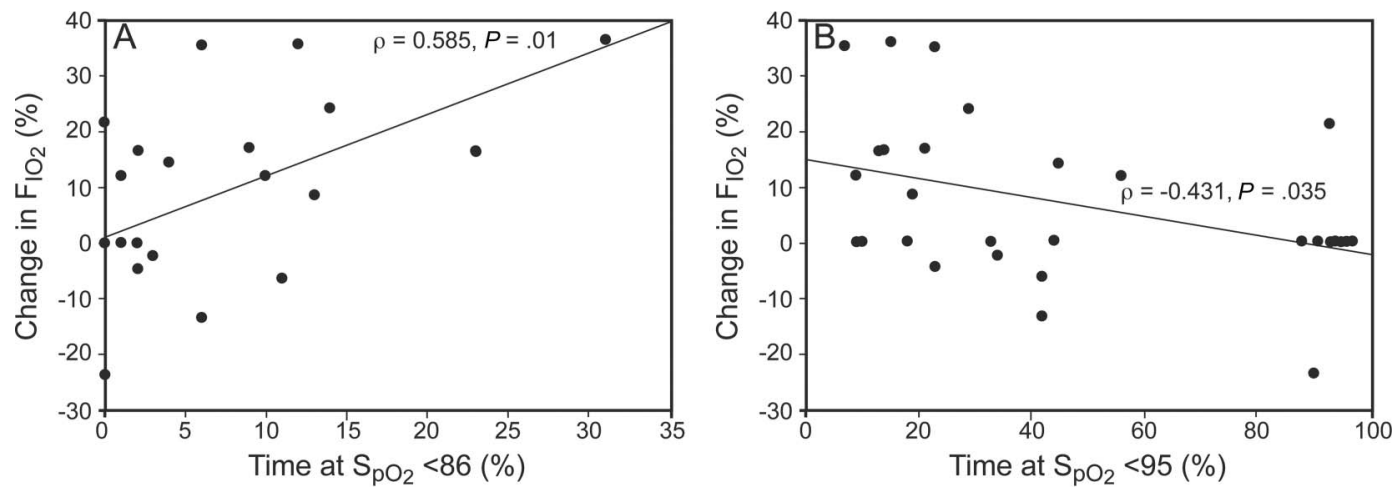

Fig. 4. Correlation between percent change in $\mathrm{F}_{\mathrm{IO}_{2}}$ post-transition and percentage of time with $\mathrm{S}_{\mathrm{pO}_{2}}<86 \%$ (A) and percentage of time with $\mathrm{SpO} 2>95 \%$ (B). For each subject, the percentage change in $\mathrm{F}_{\mathrm{IO}_{2}}$ post-transition was calculated. The Spearman's rank correlation test was used to calculate the correlation coefficient between percent change in $\mathrm{F}_{\mathrm{IO}_{2}}$ and percentage of time with $\mathrm{S}_{\mathrm{pO}_{2}}<86 \%$ and $\mathrm{S}_{\mathrm{pO}_{2}}>95 \%$ for the 24-h periods pre- and post-transition.

compared with prophylactic or early surfactant therapy studies, early CPAP results in lower rates of bronchopulmonary dysplasia/death. ${ }^{18,21,23}$ HFNC has been in- creasingly used to provide primary respiratory therapy initiated at birth, as postextubation support, or transitioning from CPAP. However, large randomized trials 
have not been performed comparing efficacy of HFNC with that of nasal CPAP. ${ }^{19}$

Because methods of weaning CPAP vary in clinical practice, several studies have evaluated alternative CPAP weaning protocols. ${ }^{24-27}$ In a multi-center, randomized control trial comparing 3 CPAP weaning protocols, Todd et $\mathrm{al}^{24}$ demonstrated that decreasing CPAP pressure rather than cycling time off CPAP is the most effective method for CPAP weaning. ${ }^{24}$ The group "taken off CPAP with the view to stay off" had significantly shortened CPAP weaning time, oxygen duration, incidence of bronchopulmonary dysplasia, and duration of hospitalization compared with cycling on/off CPAP with incremental time off CPAP with or without low-flow nasal cannula support when off CPAP. In this current study, all subjects were transitioned off CPAP of $+4-5 \mathrm{~cm} \mathrm{H}_{2} \mathrm{O}$ or HFNC of $2 \mathrm{~L} / \mathrm{min}$ with the intention to remain off support.

In the trial by Todd et al, ${ }^{24}$ infants were randomized to a weaning method once stability criteria were reached. The stability criteria included CPAP of $4-6 \mathrm{~cm} \mathrm{H}_{2} \mathrm{O}, \mathrm{F}_{\mathrm{IO}_{2}}<0.25$ and not increasing; no significant chest retractions; $<3$ episodes of self reverting apnea and/or bradycardic and/or desaturation episodes/h for the previous $6 \mathrm{~h}$; not currently treated for PDA or sepsis; tolerating time off CPAP during care; and an average $\mathrm{S}_{\mathrm{pO}_{2}}>86 \%$ most of the time or $\mathrm{P}_{\mathrm{aO}}$ /transcutaneous $\mathrm{P}_{\mathrm{aO}_{2}}>45 \mathrm{~mm} \mathrm{Hg} \cdot{ }^{24}$ Subjects in our study met these stability criteria, but we assessed whether adding the specific criterion of percentage of time with $\mathrm{S}_{\mathrm{pO}_{2}}<86 \%$ in the 24-h period before anticipated respiratory support wean better predicted transition failure. The high specificity of the $\mathrm{S}_{\mathrm{pO}_{2}}<86 \%>15 \%$ of time cut-off by the receiver operating characteristic analysis suggests that infants who experience $\mathrm{S}_{\mathrm{pO}_{2}}<86 \%$ for $<15 \%$ of time pre-transition are likely to successfully transition from noninvasive positive pressure support. In addition, we observed that infants who were successfully weaned spent a greater percentage of time with $\mathrm{S}_{\mathrm{pO}_{2}}>95 \%$ than the wean failure group, suggesting that these infants may have been successfully weaned earlier and avoided hyperoxia exposure. The $\mathrm{S}_{\mathrm{pO}_{2}}$ histogram also predicted post-transitional changes in oxygen requirements. With increasing percentage of time with $\mathrm{S}_{\mathrm{pO}_{2}}<86 \%$ pre-transition, there was an increase in $\mathrm{F}_{\mathrm{IO}_{2}}$ post-transition, and increasing percentage of time with $\mathrm{S}_{\mathrm{pO}_{2}}>95 \%$ was associated with reduced $\mathrm{F}_{\mathrm{IO}_{2}}$ post-transition.

There are a number of limitations of our study. Due to the observational nature of the study, decisions concerning timing to transition off CPAP/HFNC and reinstituting respiratory support were made by the clinical team using neonatal ICU guidelines rather than by prespecified study criteria. The clinical team preference determined whether CPAP or HFNC was used for noninvasive respiratory support. Although these modes may improve gas exchange by different mechanisms, similar compliance and work of breathing have been noted in prior crossover studies of a range of CPAP/HFNC support. ${ }^{30,31}$ In this study, there were no differences in $\mathrm{S}_{\mathrm{pO}_{2}}$ histogram distributions between CPAP and HFNC groups pre- and post-wean. In addition, there was overlap of the highest histogram quintile $(>95 \%)$ of the Nellcor oximeter and the target $\mathrm{O}_{2}$ saturation range for the $\geq 33$ weeks postmenstrual age (9097\%). This is an unavoidable limitation of our study, since the oximeter ranges are fixed, and the target ranges are set by clinical practice. However, predicting wean failure as percentage of time spent with $\mathrm{S}_{\mathrm{pO}_{2}}<86 \%$ avoids this limitation, since there is agreement with the lower 2 quintiles $(<80 \%$ and $81-85 \%)$ for the target oxygen saturations for infants $<33$ weeks and $\geq 33$ weeks postmenstrual age. Although there was a greater difference between the selected $\mathrm{S}_{\mathrm{pO}_{2}}<86 \%$ cut-off and the lower $\mathrm{S}_{\mathrm{pO}_{2}}$ target for infants $\geq 33$ weeks $(90 \%)$ and $<33$ weeks postmenstrual age $(88 \%)$, we could not determine the impact of this difference on the predictive value of time $<86 \% \mathrm{~S}_{\mathrm{pO}_{2}}$, since the histogram distributions were similar for the infants in the different $\mathrm{S}_{\mathrm{pO}_{2}}$ target ranges. The clinical teams could view the real-time $\mathrm{S}_{\mathrm{pO}_{2}}$ but not the downloaded 24-h histograms, and this information was not incorporated into clinical care decisions.

\section{Conclusions}

In summary, the current study suggests that the pulse oximetry histogram combined with clinical stability criteria may better determine readiness for transitioning off CPAP/HFNC with an ultimate goal of preventing transition failures. Future trials of stability criteria incorporating $\mathrm{S}_{\mathrm{pO}_{2}}$ histogram data are warranted to better identify readiness for noninvasive respiratory support transition.

\section{REFERENCES}

1. BOOST II United Kingdom Collaborative Group, BOOST II Australia Collaborative Group, BOOST II New Zealand Collaborative Group, Stenson BJ, Tarnow-Mordi WO, Darlow BA, et al. Oxygen saturation and outcomes in preterm infants. N Engl J Med 2013; 368(22):2094-2104.

2. SUPPORT Study Group of the Eunice Kennedy Shriver NICHD Neonatal Research Network, Carlo WA, Finer NN, Walsh MC, Rich W, Gantz MG, et al. Target ranges of oxygen saturation in extremely preterm infants. N Engl J Med 2010;362(21):1959-1969.

3. Di Fiore JM, Bloom JN, Orge F, Schutt A, Schluchter M, Cheruvu $\mathrm{VK}$, et al. A higher incidence of intermittent hypoxemic episodes is associated with severe retinopathy of prematurity. J Pediatr 2010; 157(1):69-73.

4. Martin RJ, Wang K, Köroğlu O, Di Fiore J, Kc P. Intermittent hypoxic episodes in preterm infants: do they matter? Neonatology 2011;100(3):303-310.

5. Chen ML, Guo L, Smith LE, Dammann CE, Dammann O. High or low oxygen saturation and severe retinopathy of prematurity: a metaanalysis. Pediatrics 2010;125(6):e1483-e1492.

6. Stenson BJ. Oxygen targets for preterm infants. Neonatology 2013; 103(4):341-345. 
7. Tin W, Milligan DW, Pennefather P, Hey E. Pulse oximetry, severe retinopathy, and outcome at one year in babies of less than 28 weeks gestation. Arch Dis Child Fetal Neonatal Ed 2001;84(2):F106-F110.

8. Ruiz TL, Trzaski JM, Sink DW, Hagadorn JI. Transcribed oxygen saturation vs oximeter recordings in very low birth weight infants. J Perinatol 2014;34(2):130-135.

9. Workie FA, Rais-Bahrami K, Short BL. Clinical use of new-generation pulse oximeters in the neonatal intensive care unit. Am J Perinatol 2005;22(7):357-360.

10. Hay WW, Jr, Brockway JM, Eyzaguirre M. Neonatal pulse oximetry: accuracy and reliability. Pediatrics 1989;83(5):717-722.

11. Fouzas S, Priftis KN, Anthracopoulos MB. Pulse oximetry in pediatric practice. Pediatrics 2011;128(4):740-752.

12. Hay WW, Jr, Rodden DJ, Collins SM, Melara DL, Hale KA, Fashaw LM. Reliability of conventional and new pulse oximetry in neonatal patients. J Perinatol 2002;22(5):360-366.

13. Bizzarro MJ, Li FY, Katz K, Shabanova V, Ehrenkranz RA, Bhandari $\mathrm{V}$. Temporal quantification of oxygen saturation ranges: an effort to reduce hyperoxia in the neonatal intensive care unit. $\mathrm{J}$ Perinatol 2014;34(1):33-38.

14. Mills BA, Davis PG, Donath SM, Clucas LM, Doyle LW. Improving compliance with pulse oximetry alarm limits for very preterm infants? J Paediatr Child Health 2010;46(5):255-258.

15. Laptook AR, Salhab W, Allen J, Saha S, Walsh M. Pulse oximetry in very low birth weight infants: can oxygen saturation be maintained in the desired range? J Perinatol 2006;26(6):337-341.

16. Hagadorn JI, Furey AM, Nghiem TH, Schmid CH, Phelps DL, Pillers DA, et al. Achieved versus intended pulse oximeter saturation in infants born less than 28 weeks' gestation: the AVIOx study. Pediatrics 2006;118(4):1574-1582.

17. Lim K, Wheeler KI, Gale TJ, Jackson HD, Kihlstrand JF, Sand C, et al. Oxygen saturation targeting in preterm infants receiving continuous positive airway pressure. J Pediatr 2014;164(4):730-736.e1.

18. Dunn MS, Kaempf J, de Klerk A, de Klerk R, Reilly M, Howard D, et al. Randomized trial comparing 3 approaches to the initial respiratory management of preterm neonates. Pediatrics 2011;128(5): e1069-e1076.

19. Manley BJ, Dold SK, Davis PG, Roehr CC. High-flow nasal cannulae for respiratory support of preterm infants: a review of the evidence. Neonatology 2012;102(4):300-308.

20. Morley CJ, Davis PG, Doyle LW, Brion LP, Hascoet JM, Carlin JB, COIN Trial Investigators. Nasal CPAP or intubation at birth for very preterm infants. N Engl J Med 2008;358(7):700-708.
21. Schmölzer GM, Kumar M, Pichler G, Aziz K, O’Reilly M, Cheung PY. Non-invasive versus invasive respiratory support in preterm infants at birth: systematic review and meta-analysis. BMJ 2013; 347:f5980.

22. Shoemaker M, Pierce M, Yoder B, DiGeronimo R. High flow nasal cannula versus nasal CPAP for neonatal respiratory disease: a retrospective study. J Perinatol 2007;27(2):85-91.

23. SUPPORT Study Group of the Eunice Kennedy Shriver NICHD Neonatal Research Network, Finer NN, Carlo WA, Walsh MC, Rich W, Gantz MG, et al. Early CPAP versus surfactant in extremely preterm infants. N Engl J Med 2010;362(21):19701979.

24. Todd DA, Wright A, Broom M, Chauhan M, Meskell S, Cameron C, et al. Methods of weaning preterm babies $<30$ weeks gestation off CPAP: a multicentre randomised controlled trial. Arch Dis Child Fetal Neonatal Ed 2012;97(4):F236-F240.

25. Fernandez-Alvarez JR, Gandhi RS, Amess P, Mahoney L, Watkins R, Rabe H. Heated humidified high-flow nasal cannula versus low-flow nasal cannula as weaning mode from nasal CPAP in infants $\leq 28$ weeks of gestation. Eur J Pediatr 2014;173(1): 93-98.

26. Jardine LA, Inglis GD, Davies MW. Strategies for the withdrawal of nasal continuous positive airway pressure (NCPAP) in preterm infants. Cochrane Database Syst Rev 2011;(2):CD006979.

27. Rastogi S, Wong W, Gupta A, Bhutada A, Deepa Rastogi, Maimonides Neonatal G. Gradual versus sudden weaning from nasal CPAP in preterm infants: a pilot randomized controlled trial. Respir Care 2013;58(3):511-516.

28. Heller SR, Cryer PE. Reduced neuroendocrine and symptomatic responses to subsequent hypoglycemia after 1 episode of hypoglycemia in nondiabetic humans. Diabetes 1991;40(2):223-226.

29. Askie LM, Henderson-Smart DJ, Irwig L, Simpson JM. Oxygensaturation targets and outcomes in extremely preterm infants. N Engl J Med 2003;349(10):959-967.

30. de Jongh BE, Locke R, Mackley A, Emberger J, Bostick D, Stefano $\mathrm{J}$, et al. Work of breathing indices in infants with respiratory insufficiency receiving high-flow nasal cannula and nasal continuous positive airway pressure. J Perinatol 2014;34(1):27-32.

31. Saslow JG, Aghai ZH, Nakhla TA, Hart JJ, Lawrysh R, Stahl GE, Pyon KH. Work of breathing using high-flow nasal cannula in preterm infants. J Perinatol 2006;26(8):476-480.

This article is approved for Continuing Respiratory Care Education credit. For information and to obtain your CRCE

(free to AARC members) visit www.rcjournal.com

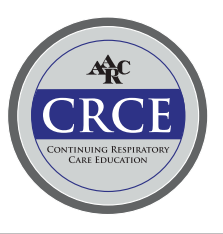

